\title{
South African guidelines for receptor radioligand therapy (RLT) with Lu-177-PSMA in prostate cancer
}

\author{
M Vorster, ${ }^{1}$ J Warwick, ${ }^{2}$ IO Lawal, ${ }^{1}$ P du Toit, ${ }^{3}$ M Vangu, ${ }^{4}$ NE Nyakale, ${ }^{5}$ R Steyn, ${ }^{6}$ AA Gutta, ${ }^{7}$ G Hart,,${ }^{8}$ S Mutambirwa, \\ A Ellmann, ${ }^{2}$ MM Sathekge ${ }^{1}$ \\ ${ }^{1}$ Department of Nuclear Medicine, University of Pretoria and Steve Biko Academic Hospital, Pretoria, South Africa \\ ${ }^{2}$ Department of Nuclear Medicine, Tygerberg Academic Hospital and Stellenbosch University, Stellenbosch, South Africa \\ ${ }^{3}$ Nuclear Medicine, Bloemfontein Mediclinic, Bloemfontein, South Africa \\ ${ }^{4}$ Department of Nuclear Medicine, Charlotte Maxeke Johannesburg Academic Hospital and University of the Witwatersrand, \\ Johannesburg, South Africa \\ ${ }^{5}$ Department of Nuclear Medicine, Inkosi Albert Luthuli Central Hospital and University of KwaZulu-Natal, Durban, South \\ Africa \\ ${ }^{6}$ Division of Nuclear Medicine, Groote Schuur Hospital and University of Cape Town, Cape Town, South Africa \\ ${ }^{7}$ Department of Nuclear Medicine, Sefako Makgatho University, Pretoria, South Africa \\ ${ }^{8}$ Oncology, Rondebosch Medical Centre, Cape Town, South Africa
}

Corresponding author: Mike M. Sathekge (mike.sathekge@up.ac.za)

The College of Nuclear Physicians of South Africa practice guidelines on peptide receptor radionuclide therapy in neuroendocrine tumours

Background: Prostate cancer is an important cause of morbidity and mortality in South Africa, as it is in the rest of the world. In African men, however, prostate cancer tends to follow a more aggressive course when compared to their European counterparts. This is attributed to a plethora of diverse factors of which an underlying genetic component has been shown to be an important aspect. Such differences highlight the need for individualised therapy and for local guidelines. The aim of this guideline is to aid nuclear physicians and other clinicians who manage patients with prostate cancer in the correct identification and treatment of patients who are likely to benefit from receptor radioligand therapy.

Recommendations: There are a multitude of treatment modalities available for the treatment of prostate cancer and these therapies may be required at various time points during the course of the disease in any individual patient. A multidisciplinary approach is crucial in deciding which therapy, or combination of therapies, would be most advantageous at particular time points. The multidisciplinary team should include a urologist, oncologist and nuclear medicine physician as a minimum, and should ideally also involve a palliative/pain specialist, a dietician and a psychologist.

Conclusion: Treatment with ${ }^{177} \mathrm{Lu}-\mathrm{PSMA}$ has emerged as a promising systemic modality, which involves the delivery of targeted radiation therapy in the form of $\beta$-particles to sites of tumour tissue. Therapy is provided on an outpatient basis, is well tolerated with relatively few side effects and has a positive effect on overall survival and quality of life. At present, it is used mostly in the setting of advanced, castrate-resistant cancer. Patients are selected (amongst other criteria) based on the prior PSMA-based SPECT/PET/CT imaging $\left({ }^{99 \mathrm{~m} T c-},{ }^{68} \mathrm{Ga}-\right.$ or $\left.{ }^{18} \mathrm{~F}-\mathrm{PSMA}\right)$, which should demonstrate sufficient receptor expression in order to consider PSMA-based targeted radionuclide therapy. Such imaging of an intended target prior to its therapeutic targeting is known as a theranostic approach.

Keywords: ${ }^{177} \mathrm{Lu}$-PSMA, RLT, metastatic castrate-resistant prostate cancer, theranostics 


\section{Purpose}

The purpose of this guideline is to aid clinicians and nuclear physicians in the identification of patients who are likely to benefit from targeted radionuclide therapy; to provide information regarding the therapy procedure, follow-up management and expected outcomes; to ensure optimal patient indication and management via a multidisciplinary team approach, and to avoid possible adverse effects that may be caused by inadvertent concurrent therapies. This practice guidance was written based on recent publications and opinions of experts routinely using this treatment modality in patient management.

\section{Definitions/Key concepts}

RLT: Radioligand therapy involves the intravenous administration of a radiopharmaceutical which emits (in this instance) a $\beta$-particle and targets a specific receptor overexpressed on cancer cells in order to deliver targeted radiation to cancer cells. ${ }^{1}$

Lu-177: Lutetium-177 is a radionuclide with $\beta$ - and a $\gamma$-emissions with a physical half-life of 6.7 days. The mean energy of the $\beta$-particle is $0.498 \mathrm{MeV}$ with a mean softtissue penetration of $0.23 \mathrm{~mm}$. The short-range $1 \mathrm{~mm}$ path length of the beta-particle emitted by ${ }^{177} \mathrm{Lu}$ enables effective delivery of radiation to tumours while minimising damage to surrounding normal tissues. The two main gamma energies of $113 \mathrm{keV}(6 \%)$ and $208 \mathrm{keV}(11 \%)$ enable post-therapy imaging. Imaging ensures that the therapy has been delivered to the intended areas of disease and also allows for response evaluation with subsequent cycles. ${ }^{2}$

PSMA: Prostate specific membrane antigen is a type II transmembrane glycol-protein with enzymatic properties (also known as folate hydrolase I), which is anchored in the cell membrane of prostate epithelial cells. PSMA is overexpressed by 100 to 1000 times in prostate cancers, with expression further increased in metastatic and castration-resistant carcinomas. It is used in various forms such as PSMA-11 for imaging, PSMA-617 for therapy and PSMA I\&T, which may be used in both imaging and therapy. ${ }^{3}$

Theranostics: This is an approach that combines both diagnostic and therapeutic elements whereby the expression of a targeted receptor has to be visualised with a suitable imaging modality prior to consideration of such treatment.

Castrate-resistant prostate cancer (CRPC) is defined as a testosterone level of $<50 \mathrm{ng} / \mathrm{dl}$ in addition to any of the following:

1. Biochemical progression: this is defined as three consecutive rises in PSA of a week apart, which results in two values of $50 \%$ greater than the nadir and a PSA $>2 \mathrm{ng} / \mathrm{ml}$.

2. Radiological progression: any new lesions on appropriate imaging. ${ }^{4}$

\section{Background}

Prostate cancer remains one of the leading causes of mortality amongst men globally with a median incidence rate of $19.5 / 100000$, which increases with age to reach a peak incidence over the age of 65 years. Despite the lack of reliable cancer registries in Africa, it is believed that the numbers are similar. However, there is a well-documented body of evidence, which suggests that prostate cancer follows a far more aggressive course in African men when compared to European men. African men are 1.6 times more likely to develop prostate cancer when compared to their European counterparts and twice as likely to die from this disease. This disparity is more pronounced than in any other malignancy and has in the past been attributed to factors such as socioeconomic status, access to health care and screening, diet, cultural factors and genetic differences. Convincing recent evidence, however, points to biological, molecular and genetic differences as the major role players in the observed differences. ${ }^{5}$

Sathekge et al. recently compared the imaging findings on ${ }^{68} \mathrm{Ga}$-PSMA in black patients to those of white patients and demonstrated, firstly, that SUVmax and s-PSA were significantly related to the Gleason score and secondly, that these values were significantly higher in black patients versus white patients, suggesting a more aggressive course in the former. ${ }^{6}$

In the setting of metastatic castrate-resistant prostate cancer (mCRPC), several third-line systemic drugs (often combined with steroids) have demonstrated an improvement in overall survival (OS). These drugs include Abiraterone, Enzalutamide, Cabazitaxel, Sipoleucel and Radium-223 and are often prescribed once treatment with Docetaxel has failed. There are, however, significant adverse effects associated with the majority of these drugs. ${ }^{7-12}$

Recently, radioligand therapy (RLT) with ${ }^{177} \mathrm{Lu}$-PSMA has emerged as a promising alternative to the aforementioned therapies. This radiopharmaceutical targets PSMA to deliver relatively selective radiation in the form of $\beta$-particles to sites of prostate cancer involvement. Lutetium-177 is a radionuclide with $\beta$ - and a $\gamma$-emissions with a physical halflife of 6.7 days. The mean energy of the $\beta$-particle is 0.498 $\mathrm{MeV}$ and it has a mean soft-tissue penetration of $0.23 \mathrm{~mm}$. The short-range $1 \mathrm{~mm}$ path length of the beta-particle emitted by ${ }^{177} \mathrm{Lu}$ enables effective delivery of radiation to tumours while minimising damage to surrounding normal tissues. The two main gamma energies of $113 \mathrm{keV}(6 \%)$ and 208 $\mathrm{keV}(11 \%)$ enable post-therapy imaging. Imaging ensures delivery of the therapy to the intended areas of disease and also allows for response evaluation with subsequent cycles. ${ }^{2}$ Prostate specific membrane antigen (PSMA) is a type II transmembrane glycol-protein with enzymatic properties which is anchored in the cell membrane of prostate epithelial cells. PSMA is overexpressed 100 to 1000 times in prostate cancers compared to normal prostate tissue, with further increased expression in metastatic and castration-resistant carcinomas. ${ }^{3}$ Currently, however, therapy with ${ }^{177} \mathrm{Lu}-\mathrm{PSMA}$ 
is considered very late in the therapeutic sequence, once all other standard therapies have failed and is only approved and funded by a small number of funders.

A recent systematic review conducted by Von Eyben et al. indirectly compared outcomes and adverse effects of thirdline therapy with RLT in patients with metastatic CRPC. The authors concluded that RLT had better treatment effects with fewer side effects when compared to third-line therapy despite being offered much later in the therapeutic sequence. ${ }^{13}$

In the first prospective study of its kind, Hofman et al. similarly demonstrated that treatment with ${ }^{177} \mathrm{Lu}-\mathrm{PSMA}$ resulted in high response rates, a low toxicity profile and improvements in quality of life (QoL), especially with regards to pain management. ${ }^{14}$

A 2018 meta-analysis by Kim aimed to evaluate the therapeutic effects of RLT with ${ }^{177} \mathrm{Lu}$-PSMA in patients with CRPC. This meta-analysis included 10 studies with a total of 455 patients. The authors found that the most significant decline in s-PSA was likely to occur after the first cycle of ${ }^{177}$ Lu-PSMA and that any decline in s-PSA was associated with a significant survival benefit. ${ }^{15}$

Several studies from various centres across the globe have demonstrated the likely benefit of therapy with Lu-177-PSMA and randomised prospective trials are in progress. ${ }^{16-25}$

\section{The referral process}

Patient referral should take place within the framework of a multidisciplinary team (MDT), which should include urologists, oncologists (medical and radiation) and nuclear medicine specialists as a minimum. Ideally, the team should also include a dietician, palliative/pain specialist, a radiologist and a psychologist.

\section{The referring physician should provide the following information and documentation:}

a) A referral form must be completed and submitted to the MDT meeting coordinator, incorporating patient details, patient issues (physical, psychological and social) and the patient's own goals. It is necessary to detail the specific reason for the referral.

b) A consent form should be attached to the referral form.

\section{Procedural details: ${ }^{177}$ Lu-PSMA-617}

Therapy involves the systemic administration of a specific well-defined radiopharmaceutical $\quad\left({ }^{177} \mathrm{Lu}-\mathrm{PSMA}-617\right)$ consisting of a $\beta$-emitting radionuclide labelled to a peptide for the purpose of delivering cytotoxic radiation to a tumour. Radionuclide therapy is therefore a molecularly targeted radiation therapy and is thus distinct from external beam radiation therapy.

Treatment with ${ }^{177} \mathrm{Lu}$-PSMA should only be administered by a qualified nuclear medicine physician at an appropriately licenced facility with nursing staff who are trained on radiation safety and a medical physicist or radiation protection officer.

\section{Indications for PRLT}

- Patients with metastatic castrate-resistant prostate cancer (mCRPC)

- Patients who are unable or unwilling to undergo conventional therapy (e.g. chemotherapy, hormonal- and/ or radiation therapy)

- Patients with corresponding increased uptake on any of the following imaging studies: ${ }^{99 \mathrm{~m} T c-P S M A ~ S P E C T, ~}$ ${ }^{68} \mathrm{Ga}$-PSMA PET, or ${ }^{18} \mathrm{~F}$-PSMA PET

\section{Contraindications for PRLT}

\section{Absolute}

- No or insufficient tracer intensity (less intense than the liver) on appropriate PSMA-based imaging and/or significant discordant uptake on ${ }^{18} \mathrm{~F}$-FDG PET/CT

- Localised/operable disease

- Severe acute concomitant illnesses

- Severe unmanageable psychiatric disorder

\section{Relative}

- ECOG performance status of greater than 2

- Severely compromised renal function $\left(\mathrm{GFR}<40 \mathrm{ml} / \mathrm{min} / \mathrm{m}^{2}\right)$

- Urinary tract obstruction

- Severely compromised bone marrow. Suggested reference values:

$\begin{array}{ll}\text { - } & \mathrm{WBC}<3000 / \mu \mathrm{l} \\ \text { - } & \text { Pbsolute neutrophil count }<1500 / \mu \mathrm{l} \\ \text { Platelets }<75000 / \mu \mathrm{l} \\ \mathrm{Hb}<9 \mathrm{~g} / \mathrm{dl}\end{array}$

\section{Clinical information}

- The decision to treat with ${ }^{177} \mathrm{Lu}-\mathrm{PSMA}$ should be made following a MDT meeting. The team should at least include the treating urologist/oncologist as well as the nuclear medicine team.

- Referring doctor to provide the following:

- Detailed histopathology

- Results of any recent scans/biochemistry

- Treatment history (prostatectomy, chemotherapy, external beam radiation) and relevant medical history (e.g. diabetes, hypertension)

- A thorough clinical examination

- Patients should undergo PSMA-based SPECT imaging or PET/CT imaging with ${ }^{99 \mathrm{~m} T c-P S M A},{ }^{68} \mathrm{Ga}-\mathrm{PSMA} /{ }^{18} \mathrm{~F}-$ PSMA for therapy selection purposes as well as to serve as a baseline for treatment response assessment. Imaging with ${ }^{68} \mathrm{Ga}$-PSMA PET is preferable where this modality is available.

- The following criteria should be considered before RLT:

- ECOG performance status of less than 2

- Renal function evaluation by performing a baseline ${ }^{99 m}$ Tc-MAG3 renal scan and/or GFR assessment. 


\section{Patient preparation and consent}

- The nuclear physician will obtain informed consent from the patient.

- Patient must sign consent form before the therapy is ordered.

- Provide the patient with an information sheet with information on the treatment and clear advice on precautionary measures to take to minimise radiation to self and others.

- List of current medications - exclude myelosuppressive drugs.

- No need to interrupt first-line androgen deprivation treatment (however, care should be exercised if patient is on second-line androgen deprivation therapy or chemotherapy as no prospective trials are available to elicit safety).

During discussion, the following points in the consent form should be completely understood by the patient:

1. Objective and outcome of RLT

2. Possible side effects of RLT

3. Interim- and follow-up management during RLT

4. Precautionary measures that should be followed after RLT to keep radiation exposure as low as reasonably achievable

\section{${ }^{177}$ Lutetium-PSMA therapeutic procedure}

\section{Kidney protection}

- The kidney is the dose-limiting organ at the activities normally used for RLT and should therefore be protected.

- Protection is obtained with the use of an amino acid infusion before and during RLT administration.

- Hydrating the patient with normal saline may also reduce nausea and vomiting caused by amino acids.

Other:

- Corticosteroids to reduce risk of swelling and mechanical obstruction in cerebral, spinal, lung or other metastases which may cause compromise of vital organ functions.

\section{Administration}

- Doses should be administered every 6-8 weeks, for 3-6 cycles at a mean dose of $7.4 \mathrm{GBq}(5.55$ to $8 \mathrm{GBq})$.

- The number of cycles to be administered will depend on the treatment response as well as the side effect profile.

- Complete hot lab logbook on receipt of Lutetium-177 (date, batch number, activity).

- Confirm that patient followed preparation as requested.

- Measure ${ }^{177}$ Lu-PSMA dose before administration.

- Confirm quality control (QC) results documented in hot lab logbook.

- Document ${ }^{177} \mathrm{Lu}-\mathrm{PSMA}$ dose, date, time and nuclear physician who administered dose in patient file.

- The radiopharmaceutical should be diluted with saline to a volume of $10-100 \mathrm{ml}$ and be administered via an indwelling catheter as per misadministration prevention protocol over 10-30 minutes.
- The line should be flushed with saline after completion of radiopeptide infusion.

- Vital signs should be monitored before and after therapy.

- Nursing personnel should be instructed in radiation safety.

- Radiation emission to be measured with a hand-held gamma counter and patients to be discharged when below $20 \mu \mathrm{Sv}$ per hour at $1 \mathrm{~m}$.

\section{Patient management during cycles}

- Thorough clinical history and examination during each visit and completion of QoL questionnaires.

- Document any relevant patient information from patient/ referring doctor in patient file.

- Post-therapeutic ${ }^{177} \mathrm{Lu}-\mathrm{PSMA}$ scans are acquired at 4 hours, 24 hours and 48 hours after treatment infusion. These scans provide valuable information on the intensity of uptake and localisation of the tracer. They are also used to assess the treatment response to prior therapy cycles as well as in dosimetry calculations.

- Post-therapy treatment summaries as well as the results of the most recent follow-up imaging studies should be provided to the referring clinician.

- Frequent MDT meetings to be held in order to assess global therapy response, to manage any side effects and to allow for discussions regarding further or altered clinical management when needed.

- Clinical examination and blood tests should be performed at 2- to 4-week intervals for evaluation of toxicity and response. A complete blood count, s-PSA, liver function tests, and serum creatinine or clearance tests should be included in the routine bloodwork.

- RLT cycles may be extended or interrupted if blood values are lower than the minimum requirements.

\section{Long-term patient management}

- Full blood count, renal function, liver function and s-PSA should be done every 3 months for 12 months after completion of cycles.

- Imaging evaluation with ${ }^{68} \mathrm{Ga}-\mathrm{PSMA} \mathrm{PET} / \mathrm{CT} /$, ${ }^{99 \mathrm{~m} T c-}$ PSMA $/{ }^{18}$ F-PSMA PET/CT should be done 3-6 months after treatment to evaluate treatment response, depending on the clinical picture.

- Repeat Lu-177-PSMA cycles can be considered in the absence of any contraindications, and where the potential benefits clearly outweigh the risks.

\section{Treatment goals and outcomes}

\section{Primary endpoints include the following:}

- A PSA decline of greater than $50 \%$ from baseline as per the Prostate Cancer Clinical Trial Working Group criteria $^{26}$ 
- Imaging responses on post-therapeutic imaging as well as on follow-up PSMA-based SPECT/ PET/CT imaging

- An improvement in QoL (as assessed on a standardised questionnaire, e.g. EORTC-Q30 and Brief Pain)

- Minimal side effects or toxicity as per common terminology criteria for adverse events (CTCAE). ${ }^{27}$

\section{Expected outcomes and predictors of a negative response}

Several studies have demonstrated a positive effect on overall survival and progression free survival.

Likely predictors of a negative response to RLT include younger age $(<65)$, high levels of g-glutamyl transferase $>100 \mathrm{U} / \mathrm{l})$, lower pretherapeutic haemoglobin, a higher Gleason score, a higher number of platelets, high C-reactive protein $(>20 \mathrm{mg} / \mathrm{l})$, regular need for pain medication, and higher lactate dehydrogenase. ${ }^{28}$

It is important to note that up to a third of men treated to date show progressive disease noted on imaging or rising PSA despite treatment and thus treatment cycles will need to be discontinued.

\section{Other considerations}

\section{Pain management in prostate cancer}

Another important aspect to consider is the management of pain in patients with prostate cancer, which will be briefly outlined here. For a more detailed overview, readers are referred to the ESMO Clinical Practice Guidelines of April $2018 .^{29}$

Central to the management of the chronic pain often experienced in cancer patients is the principle of prescribing regular analgesics rather than on an "as needed" basis, and to start with oral analgesics wherever possible in order to optimise pain relief and limit possible side effects.

Firstly, it has to be established what the nature of the pain is, whether nociceptive due to ongoing processes of tissue damage (either visceral or somatic), or neuropathic in nature. Then, the quality of the pain has to be determined based on one of the internationally established scales, such as the Verbal Analogue Scale (VAS), the Verbal Rating Scale (VRS) or the Numerical Rating Scale (NRS). Once the level of the pain has been assessed, analgesics should be given according to the WHO analgesic ladder, which starts with paracetamol and nonsteroidal anti-inflammatory drugs (NSAIDs) at low levels of pain and recommends strong opioids at high levels of pain. Opioids tend to be the mainstay of therapy in the type of chronic pain often experienced by cancer patients. ${ }^{29}$

Commonly used "strong opioids" include morphine and fentanyl (often prescribed as transdermal administration) and oxycodone. ${ }^{30}$

In patients with renal impairment, fentanyl and buprenorphine may be the preferred drugs.

An important aspect to keep in mind in those patients who are concurrently treated with enzalutamide is that it is a potent inducer of the P-450 CYP enzyme and may therefore decrease the efficacy of certain opioids. In such cases, it may be preferable to treat with morphine or hydromorphone rather than with oxycodone, fentanyl or buprenorphine..$^{29,30}$

\section{Management of side effects}

Side effects which may occur as a result of therapy include nausea (which may be significant and has been reported in up to $10 \%$ ), fatigue (reported in up to $25 \%$ of treated patients), haematological toxicity, renal impairment (rarely reported) and xerostomia (approximately 30\%). These side effects when experienced tend to be mild and may mostly be treated conservatively.

Nausea may be effectively treated with a dopamineantagonist, such as metoclopramide where it is as a result of opioid treatment or alternatively with ondansetron. ${ }^{29}$

Haematological toxicity is the most commonly reported serious side effect related to therapy and occurs mostly as a bystander effect in those patients with significant skeletal metastases and/or borderline marrow function. In such patients, up to $10-25 \%$ experienced a Grade 1-2 reduction in haemoglobin or platelets, with no significant marrow toxicity reported in the absence of significant skeletal metastases. ${ }^{31,32}$

\section{Cost-effectiveness}

The financial implications to the individual patient may be significant as only some medical aids have approved funding for Lu-177-PSMA in selected cases. However, the costs are likely considerably less when compared to conventional forms of systemic therapy such as chemotherapy, with significantly fewer side effects. From the perspective of the medical funder, both the direct and indirect costs are likely to be significantly reduced. Four to six cycles administered on an outpatient basis compares very favourably to standard therapeutic regimens in formal cost-effectiveness studies performed in metastatic castrate-resistant prostate cancer. ${ }^{33-36}$

Several studies have demonstrated an improvement in quality of life as well as in outcome measures following RLT. Side effects occur less frequently than with standard third-line therapies and are mostly treated without the need for hospital admission. $^{13}$

\section{Dosimetry and radiation protection}

Organs that are susceptible to toxicity during RLT include the kidneys, the salivary- and lacrimal glands as well as the bone marrow. The initial critical threshold for absorbed dose to the kidney of 23 Gy has recently been amended to 28 and even up to 40 Gy depending on the presence of additional risk factors. Various groups have demonstrated far lower doses received by the kidneys with values closer to between 0.8 and $2.2 \mathrm{mGy} / \mathrm{MBq}$. Significantly higher absorbed doses of up to $7.5 \mathrm{~Gy} /$ cycle to the parotid glands have been reported. ${ }^{18,19,37-39}$

In South Africa, the patient-specific radiation doses in a patient undergoing ${ }^{177} \mathrm{Lu}-\mathrm{PSMA}$ with a standard 6-8 GBq dose falls within the range which can be administered safely and legally within an outpatient setting. The majority of ${ }^{177}$ Lu-PSMA is excreted via the kidneys in the first 48 hours following injection, and instructions need to be given to patients, staff and families on managing potential radioactive 
contamination. Patients are required to remain in the department for 2-4 hours for observation and for measured radiation levels to decrease to below $20 \mu \mathrm{Sv} / \mathrm{hr}$.

\section{Issues for future clarification}

- The need for amino acids vs. alternative forms of IV hydration (such as normal saline) in renal function protection.

- Reserving Lu-177-PSMA only for patients with metastatic castrate-resistant prostate cancer.

- Effects of combination therapy with androgen blockade.

- Combination treatment strategies which include chemotherapeutic regimens and external beam radiation therapy.

- $\quad$ Addition of steroids with potential risks and benefits.

- Minimum number of cycles to be completed and decisions on repeated RLT.

- Optimisation and individualisation of activity to be administered.

- Minimisation and treatment of the salivary gland toxicity.

- Funding.

\section{REFERENCES}

1. Ahmadzadehfar $\mathrm{H}$, Rahbar $\mathrm{K}$, Kürpig $\mathrm{S}$, Bögemann $\mathrm{M}$, Claesener M, Eppard E, Gärtner F, Rogenhofer S, Schäfers M, Essler M. Early side effects and first results of radioligand therapy with (177)Lu-DKFZ-617 PSMA of castrate-resistant metastatic prostate cancer: a two-centre study. EJNMMI Res. 2015 Dec;5(1):114. doi: 10.1186/s13550-015-0114-2.

2. Dash A, Pillai MR, Knapp FF. Production of (177)Lu for Targeted Radionuclide Therapy: Available Options. Nucl Med Mol Imaging. 2015 Jun;49(2):85-107. doi: 10.1007/s13139014-0315-z.

3. Sweat SD, Pacelli A, Murphy GP, Bostwick DG. Prostatespecific membrane antigen expression is greatest in prostate adenocarcinoma and lymph node metastases. Urology. 1998 Oct 1;52(4):637-40.

4. Heidenreich A, Bastian PJ, Bellmunt J, Bolla M, Joniau S, Van der Kwast T, et al. EAU guidelines on prostate cancer. Part II: Treatment of advanced, relapsing, and castration-resistant prostate cancer. Eur Urol. 2014 Feb 1;65(2):467-79.

5. Guo Y, Sigman DB, Borkowski A, Kyprianou N. Racial differences in prostate cancer growth: apoptosis and cell proliferation in Caucasian and African-American patients. Prostate. 2000 Feb 1;42(2):130-6.

6. Sathekge M, Lengana T, Maes A, Vorster M, Zeevaart J, Lawal I, et al. 68Ga-PSMA-11 PET/CT in primary staging of prostate carcinoma: preliminary results on differences between black and white South-Africans. Eur J Nucl Med Mol Imaging. 2018 Feb;45(2):226-234. doi: 10.1007/s00259-017-3852-8.

7. Tannock IF, De Wit R, Berry WR, Horti J, Pluzanska A, Chi $\mathrm{KN}$, et al. Docetaxel plus prednisone or mitoxantrone plus prednisone for advanced prostate cancer. New Engl J Med. 2004 Oct 7;351(15):1502-12.

8. De Bono JS, Oudard S, Ozguroglu M, Hansen S, Machiels JP, Kocak I, et al. Prednisone plus cabazitaxel or mitoxantrone for metastatic castration-resistant prostate cancer progressing after docetaxel treatment: a randomised open-label trial. Lancet. 2010 Oct 2;376(9747):1147-54. doi: 10.1016/S01406736(10)61389-X.
9. De Bono JS, Logothetis CJ, Molina A, Fizazi K, North S, Chu L, et al. Abiraterone and increased survival in metastatic prostate cancer. N Engl J Med. 2011 May 26;364(21):1995-2005. doi: 10.1056/NEJMoa1014618.

10. Scher HI, Fizazi K, Saad F, Taplin ME, Sternberg CN, Miller $\mathrm{K}$, et al. Increased survival with enzalutamide in prostate cancer after chemotherapy. New Engl J Med. 2012 Sep 27;367(13):1187-97.

11. Sartor O, Coleman R, Nilsson S, Heinrich D, Helle SI, O'Sullivan JM, et al. Effect of radium-223 dichloride on symptomatic skeletal events in patients with castrationresistant prostate cancer and bone metastases: results from a phase 3, double-blind, randomised trial. Lancet Oncol. 2014 Jun;15(7):738-46. doi: 10.1016/S1470-2045(14)70183-4.

12. Kantoff PW, Higano CS, Shore ND, Berger ER, Small EJ, Penson DF, et al. Sipuleucel-T immunotherapy for castrationresistant prostate cancer. N Engl J Med. 2010 Jul 29;363(5):41122. doi: 10.1056/NEJMoa1001294.

13. Von Eyben FE, Roviello G, Kiljunen T, Uprimny C, Virgolini I, Kairemo K, Joensuu T. Third-line treatment and 177Lu-PSMA radioligand therapy of metastatic castration-resistant prostate cancer: a systematic review. Eur J Nucl Med Mol Imaging. 2018 Mar;45(3):496-508. doi: 10.1007/s00259-017-3895-x

14. Hofman MS, Violet J, Hicks RJ, Ferdinandus J, Thang SP, Akhurst T, et al. [177Lu]-PSMA-617 radionuclide treatment in patients with metastatic castration-resistant prostate cancer (LuPSMA trial): a single-centre, single-arm, phase 2 study. Lancet Oncol. 2018 Jun;19(6):825-833. doi: 10.1016/S14702045(18)30198-0.

15. Kim YJ, Kim YI. Therapeutic responses and survival effects of 177Lu-PSMA-617 radioligand therapy in metastatic castrateresistant prostate cancer: a meta-analysis. Clin Nucl Med. 2018 Oct;43(10):728-734. doi: 10.1097/RLU.0000000000002210.

16. Ahmadzadehfar H, Eppard E, Kürpig S, Fimmers R, Yordanova A, Schlenkhoff CD, et al. Therapeutic response and side effects of repeated radioligand therapy with 177Lu-PSMA-DKFZ-617 of castrate-resistant metastatic prostate cancer. Oncotarget. 2016 Mar 15;7(11):12477-88. doi: 10.18632/oncotarget.7245.

17. Ahmadzadehfar H, Essler M, Schäfers M, Rahbar K. Radioligand therapy with $177 \mathrm{Lu}-\mathrm{PSMA}-617$ of metastatic prostate cancer has already been arrived in clinical use. Nucl Med Biol. 2016 Dec;43(12):835. doi: 10.1016/j.nucmedbio.2016.08.003.

18. Baum RP, Kulkarni HR, Schuchardt C, Singh A, Wirtz M, Wiessalla $\mathrm{S}$, et al. 177Lu-labeled prostate-specific membrane antigen radioligand therapy of metastatic castration-resistant prostate cancer: safety and efficacy. J Nucl Med. 2016 Jul;57(7):1006-13. doi: 10.2967/jnumed.115.168443.

19. Delker A, Fendler WP, Kratochwil C, Brunegraf A, Gosewisch A, Gildehaus FJ, et al. Dosimetry for (177)Lu-DKFZPSMA-617: a new radiopharmaceutical for the treatment of metastatic prostate cancer. Eur J Nucl Med Mol Imaging. 2016 Jan;43(1):42-51. doi: 10.1007/s00259-015-3174-7.

20. Fendler WP, Kratochwil C, Ahmadzadehfar H, Rahbar K, Baum RP, Schmidt M, et al. 177Lu-PSMA-617 therapy, dosimetry and follow-up in patients with metastatic castration-resistant prostate cancer. Nuklearmedizin. 2016 Jun 28;55(3):123-8

21. Heck MM, Retz M, Tauber R, Knorr K, Kratochwil C, Eiber M. PSMA-targeted radioligand therapy in prostate cancer. Urologe A. 2017 Jan;56(1):32-39. doi: 10.1007/s00120-016-0274-3.

22. Kratochwil C, Giesel FL, Eder M, Afshar-Oromieh A, Benesová $\mathrm{M}$, Mier W, et al. [177 Lu] Lutetium-labelled PSMA ligandinduced remission in a patient with metastatic prostate cancer. Eur J Nucl Med Mol Imaging. 2015 May;42(6):987-8. doi: 
10.1007/s00259-014-2978-1. Epub 2015 Jan 9.

23. Rahbar K, Schmidt M, Heinzel A, Eppard E, Bode A, Yordanova A, et al. Response and tolerability of a single dose of $177 \mathrm{Lu}-$ PSMA-617 in patients with metastatic castration-resistant prostate cancer: a multicenter retrospective analysis. J Nucl Med. 2016 Sep;57(9):1334-8. doi: 10.2967/jnumed.116.173757.

24. Tagawa ST, Milowsky MI, Morris M, Vallabhajosula S, Christos P, Akhtar NH, et al. Phase II study of lutetium-177-labeled antiprostate-specific membrane antigen monoclonal antibody J591 for metastatic castration-resistant prostate cancer. Clin Cancer Res. 2013 Sep 15;19(18):5182-91. doi: 10.1158/1078-0432. CCR-13-0231.

25. Vallabhajosula S, Nikolopoulou A, Jhanwar YS, Kaur G, Tagawa ST, Nanus DM, et al. Radioimmunotherapy of metastatic prostate cancer with $177 \mathrm{Lu}$-DOTAhuJ591 anti prostate specific membrane antigen specific monoclonal antibody. Curr Radiopharm. 2016;9(1):44-53.

26. Scher HI, Halabi S, Tannock I, Morris M, Sternberg CN, Carducci MA, et al. Design and end points of clinical trials for patients with progressive prostate cancer and castrate levels of testosterone: recommendations of the Prostate Cancer Clinical Trials Working Group. J Clin Oncol. 2008 Mar 1;26(7):114859. doi: 10.1200/JCO.2007.12.4487.

27. US Department of Health and Human Services. Common terminology criteria for adverse events (CTCAE) version 4.0. National Institutes of Health, National Cancer Institute. 2009 May 28;4(03).

28. Ferdinandus J, Eppard E, Gaertner FC, Kürpig S, Fimmers $\mathrm{R}$, Yordanova A, et al. Predictors of response to radioligand therapy of metastatic castrate-resistant prostate cancer with 177Lu-PSMA-617. J Nucl Med. 2017 Feb;58(2):312-319. doi: 10.2967/jnumed.116.178228.

29. Fallon M, Giusti R, Aielli F, Hoskin P, Rolke R, Sharma M, Ripamonti CI, ESMO Guidelines Committee. Management of cancer pain in adult patients: ESMO Clinical Practice Guidelines. Ann Oncol. 2018 Oct 1;29(Suppl 4):iv166-iv191. doi: 10.1093/annonc/mdy152.

30. Westdorp H, Kuip EJM, Van Oort IM, Kramers C, Gerritsen WR, Vissers KCP. Difficulties in Pain Management Using Oxycodone and Fentanyl in Enzalutamide-Treated Patients With Advanced Prostate Cancer. J Pain Symptom Manage. 2018 Apr;55(4):e6-e8. doi: 10.1016/j.jpainsymman.2017.11.016.

31. Baum RP, Kulkarni HR, Schuchardt C, Singh A, Wirtz M,
Wiessalla S, et al. Lutetium-177 PSMA radioligand therapy of metastatic castration-resistant prostate cancer: safety and efficacy. J Nucl Med. 2016 Jan; 57(7). doi 10.2967/ jnumed.115.168443

32. Emmett L, Willowson K, Violet J, Shin J, Blanksby A, Lee J. Lutetium 177 PSMA radionuclide therapy for men with prostate cancer: a review of the current literature and discussion of practical aspects of therapy. J Med Radiat Sci. 2017 Mar;64(1):52-60. doi: 10.1002/jmrs.227.

33. Nguyen C, Lairson DR, Swartz MD, Du XL. Cost-Effectiveness of Adding Androgen Deprivation Therapy to Radiation Therapy for Men with Advanced Prostate Cancer from a US Payer's Perspective. J Manag Care Spec Pharm. 2019 Feb;25(2):225234. doi: 10.18553/jmcp.2019.25.2.225.

34. Pollard ME, Moskowitz AJ, Diefenbach MA, Hall SJ. Costeffectiveness analysis of treatments for metastatic castration resistant prostate cancer. Asian J Urol. 2017 Jan;4(1):37-43. doi: 10.1016/j.ajur.2016.11.005.

35. Grochtdreis T, König HH, Dobruschkin A, Von Amsberg G, Dams J. Cost-effectiveness analyses and cost analyses in castration-resistant prostate cancer: A systematic review. PLoS One. 2018 Dec 5;13(12):e0208063. doi: 10.1371/journal. pone. 0208063 .

36. Norum J, Nieder C. Treatments for metastatic prostate cancer (mPC): a review of costing evidence. Pharmacoeconomics. 2017 Dec;35(12):1223-1236. doi: 10.1007/s40273-017-0555-8.

37. Cremonesi M, Ferrari M, Di Dia A, Botta F, De Cicco C, Bodei L, Paganelli G. Recent issues on dosimetry and radiobiology for peptide receptor radionuclide therapy. J Nucl Med Mol Imaging. 2011 Apr 1;55(2):155-67.

38. Ljungberg $\mathrm{M}$, Celler A, Konijnenberg MW, Eckerman KF, Dewaraja YK, Sjögreen-Gleisner K, et al. MIRD pamphlet no. 26: Joint EANM/MIRD guidelines for quantitative 177Lu SPECT applied for dosimetry of radiopharmaceutical therapy. J Nucl Med. 2016 Jan;57(1):151-62. doi: 10.2967/ jnumed.115.159012.

39. Bodei L, Cremonesi M, Ferrari M, Pacifici M, Grana CM, Bartolomei M, et al. Long-term evaluation of renal toxicity after peptide receptor radionuclide therapy with 90Y-DOTATOC and 177Lu-DOTATATE: the role of associated risk factors. Eur J Nucl Med Mol Imaging. 2008 Oct;35(10):1847-56. doi: 10.1007/s00259-008-0778-1. 